\title{
Lunar Ascent Nominal Trajectory Optimization based on UKF Parameter Estimation
}

\author{
Xiang Chen ${ }^{1, a}$, Fengbo Wang ${ }^{2, b}$ and Changhong Dong ${ }^{1, c}$ \\ ${ }^{1}$ School of Astronautics, Beihang University, Beijing, China \\ ${ }^{2}$ Beijing Institute of Space Long March Vehicle, Beijing, China \\ achenxiang_buaa@163.com, bwangfengbohao@163.com, crainbowdong@buaa.edu.cn
}

Keywords: lunar ascent; nominal trajectory; two-point boundary value problem; unscented Kalman filter; parameter estimation

\begin{abstract}
Focused on the optimization of lunar ascent nominal trajectory, an algorithm based on the unscented Kalman filter (UKF) parameter estimation is proposed in this paper. The optimization of lunar ascent nominal trajectory problem requiring minimum fuel is formulated as a two-point boundary value problem (TPBVP) through the maximum principle. By treating the initial values of the costate variables as parameters to be estimated and setting the deviation of terminal values as target observations, the TPBVP is then transformed into a parameter estimation problem and solved by the UKF parameter estimation algorithm. Numerical simulation results demonstrate that the algorithm can be used to optimize lunar ascent nominal trajectory, it can also overcome the difficulty of guessing the initial values of the costate variables, and the calculating efficiency is higher than traditional optimization algorithm under the premise of guaranteed accuracy.
\end{abstract}

\section{Introduction}

Lunar ascent problem is a key technology for returning from the Moon. At present, the methods for solving lunar ascent trajectory problem are usually classified as direct method and indirect method[1]. Direct method converts the trajectory optimal problem into a nonlinear programming problem (NLP) and then obtaining the optimal solution by parameters optimization algorithms[2,3]. All the direct methods have common defects that they depend on the solving ability of for NLP software.

Indirect method uses the Pontryagin Maximum Principle and the classical variational method of Lagrange multiplier method to convert optimization problem to a TPBVP. Shooting method is widely used to solve TPBVP[4]. However, this method is too sensitive to the initial values of the costate variables, and it's difficult to guess the values because they have no physical meaning. So it's hard to be solved by the traditional variational method.

UKF is a filter suitable for nonlinear system. The advantage of UKF is that the algorithm estimates mean and covariance of the system state is more accurate and avoids linearization error by using nonlinear model directly. As a result, UKF is widely used in reentry target tracking[5], low-thrust orbit transfer[6], et al.

In this paper, an algorithm based on the UKF parameter estimation is proposed to solve the nominal trajectory optimization of lunar ascent problem requiring minimum fuel. And several simulations are carried out to illustrate the advantages of this algorithm.

\section{Lunar Ascent Nominal Trajectory Optimization}

System Modeling. Lunar ascent can be divided into 3 phases[7]. The first one is vertical rise phase, in which the ascent module gets a sufficient altitude to allow clearing of local terrain. The second one is attitude adjustment phase which lasts about 10 seconds and prepares for the next phase. The third one is powered propulsion phase, in which the ascent module rises to the predetermined position under some kind of guidance law. 
Considering that the ascent process time is short (about 5-7 minutes) so the rotation of the Moon has less influence to it, and in most conditions the ascent process starts in a coplanar orbit, besides, the main purpose of this paper is presenting a new algorithm, so the dynamic model is set up in the two-dimensional plane polar coordinates.

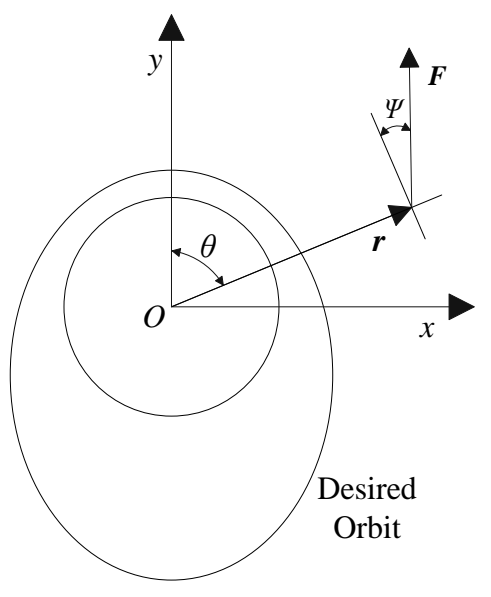

Figure 1 Lunar ascent coordinate system

As shown in Fig. 1, the origin $O$ is located in the center of the Moon, y-axis points to the pericynthion of the desired orbit, $\mathrm{x}$-axis points to the motion direction of ascent module., $\boldsymbol{r}$ is the radius vector of the ascent module to the center of the Moon, $\theta$ is the flight polar angle, $\boldsymbol{F}$ is the thrust of main engine, and $\Psi$ is the thrust angle.

The dynamic equations of lunar ascent module can be written as follows.

$$
\left\{\begin{array}{l}
\dot{\boldsymbol{r}}=\boldsymbol{v} \\
\dot{\boldsymbol{v}}=\frac{\boldsymbol{F}}{m} \sin \Psi-\frac{\mu}{\boldsymbol{r}^{2}}+\boldsymbol{r} \boldsymbol{\omega}^{2} \\
\dot{\boldsymbol{\theta}}=\boldsymbol{\omega} \\
\dot{\boldsymbol{\omega}}=-\frac{(\boldsymbol{F} / m) \cos \Psi+2 \boldsymbol{v} \boldsymbol{\omega}}{\boldsymbol{r}} \\
\dot{\boldsymbol{m}}=-\frac{\boldsymbol{F}}{I_{\mathrm{sp}}}
\end{array}\right.
$$

Where $m$ is the mass of the ascent module, $\mu$ is the gravitational parameter for the Moon, $v$ is the velocity alone the $\boldsymbol{r}$-direction, $\boldsymbol{\omega}$ is the angular velocity of $\theta, I_{\mathrm{sp}}$ is the specific impulse of the main engine, and $\boldsymbol{F}$ is set to constant thrust.

Define $\boldsymbol{x}=[\boldsymbol{r}, \boldsymbol{v}, \theta, \boldsymbol{\omega}, m]^{\mathrm{T}}$ as state parameters and $\boldsymbol{u}=\Psi$ as control parameter, the Eq. 1 then becomes

$$
\dot{\boldsymbol{x}}=f(\boldsymbol{x}, \boldsymbol{u})
$$

Minimum Fuel Consumption Problem. The essence of the Lunar ascent nominal trajectory design is to find an optimal control $\boldsymbol{u}$ which transfer the ascent module to the predetermined orbit in minimum fuel consumption. The performance functional can be defined as

$$
J=\int_{0}^{t} \dot{m}(t) d t=-\left[m\left(t_{0}\right)-m\left(t_{\mathrm{f}}\right)\right]
$$

Where $t_{0}$ is initial time and $t_{\mathrm{f}}$ is terminal time which is free. 
The initial states of lunar ascent are determined by launch conditions, and the terminal states subject to the orbit insertion conditions. Thus the boundary conditions can be written as follows.

Initial states,

$$
\boldsymbol{r}\left(t_{0}\right)=\boldsymbol{r}_{0}, \boldsymbol{v}\left(t_{0}\right)=\boldsymbol{v}_{0}, \theta\left(t_{0}\right)=\theta_{0}, \boldsymbol{\omega}\left(t_{0}\right)=\boldsymbol{\omega}_{0}, m\left(t_{0}\right)=m_{0}
$$

Terminal states,

$$
\boldsymbol{r}\left(t_{\mathrm{f}}\right)=\boldsymbol{r}_{\mathrm{f}}, \boldsymbol{v}\left(t_{\mathrm{f}}\right)=\boldsymbol{v}_{\mathrm{f}}, \boldsymbol{\omega}\left(t_{\mathrm{f}}\right)=\omega_{\mathrm{f}}
$$

TPBVP. There are 5 state variables in above system, so the costate variables can be defined as

$$
\lambda=\left[\lambda_{r}, \lambda_{v}, \lambda_{\theta}, \lambda_{\omega}, \lambda_{m}\right]^{\mathrm{T}}
$$

The Hamiltonian function is

$$
\boldsymbol{H}(\boldsymbol{x}, \boldsymbol{\lambda}, \boldsymbol{u})=\lambda^{T} f(\boldsymbol{x}, \boldsymbol{u})-\frac{\boldsymbol{F}}{I_{\mathrm{sp}}}
$$

The costate function is

$$
\dot{\lambda}=-\frac{\partial \boldsymbol{H}(\boldsymbol{x}, \boldsymbol{\lambda}, \boldsymbol{u})}{\partial \boldsymbol{x}}
$$

The transversality condition is

$$
\lambda_{\mathrm{f}}=\left[\frac{\partial \boldsymbol{M}\left(\boldsymbol{x}_{\mathrm{f}}\right)}{\partial \boldsymbol{x}}\right]^{\mathrm{T}} \boldsymbol{\xi}
$$

Where $\boldsymbol{M}\left(\boldsymbol{x}_{\mathrm{f}}\right)$ is terminal conditions, $\boldsymbol{\xi}$ is the Lagrange multiplier.

When the input control parameter is optimal, that is $\boldsymbol{u}(t)=\boldsymbol{u}^{*}(t)$, the Hamilton function reach the absolute maximum value, the extreme conditions can be written as

$$
\frac{\partial \boldsymbol{H}(\boldsymbol{x}, \boldsymbol{\lambda}, \boldsymbol{u})}{\partial \boldsymbol{u}}=0
$$

The optimal control law is

$$
\Psi=-\arctan \left(\frac{\lambda_{V} r}{\lambda_{\omega}}\right)
$$

Substitute Eq. 1 and Eq. 9, the derivation of $\Psi$ is

$$
\psi^{\prime}=\frac{-\boldsymbol{r} \lambda_{v} \lambda_{\theta}+\boldsymbol{r} \lambda_{r} \lambda_{\omega}+(2+\boldsymbol{v}) \lambda_{V} \lambda_{\omega}-2 \boldsymbol{r}^{2} \lambda_{V}^{2} \boldsymbol{\omega}-2 \lambda_{\omega}^{2} \omega}{\boldsymbol{r}^{2} \lambda_{v}^{2}+\lambda_{\omega}^{2}}
$$

Consider the range and variation rate of the thrust angle, we can adopt the optimal control law as follows 


$$
\begin{aligned}
& \Psi= \begin{cases}\Psi_{i}=\Psi_{i-1}+\Psi^{\prime *} \Delta t & \text { if } \Psi_{i} \leq \Psi_{\max } \\
\Psi_{\max } & \text { if } \Psi_{i}>\Psi_{\max }\end{cases} \\
& \Psi^{\prime}= \begin{cases}\Psi^{\prime}=\left[-\boldsymbol{r} \lambda_{v} \lambda_{\theta}+\boldsymbol{r} \lambda_{r} \lambda_{\omega}+(2+\boldsymbol{v}) \lambda_{v} \lambda_{\omega}\right. \\
\left.\quad-2 \boldsymbol{r}^{2} \lambda_{v}^{2} \boldsymbol{\omega}-2 \lambda_{\omega}^{2} \omega\right] / \boldsymbol{r}^{2} \lambda_{v}^{2}+\lambda_{\omega}^{2} & \text { if } \Psi^{\prime} \leq \Psi_{\text {max }}^{\prime} \\
\Psi_{\text {max }}^{\prime} & \text { if } \Psi^{\prime}>\Psi_{\text {max }}^{\prime}\end{cases}
\end{aligned}
$$

Where $\Psi_{\max }$ is the maximum thrust angle, and $\Psi^{\prime}{ }_{\max }$ is the maximum variation rate of the trust angle.

Substitute the optimal control law into state and costate equations, we can get the lunar ascent nominal trajectory by integrating them. Finally, the optimal control problem is transformed into a TPBVP. In the next section, We'll take use of the UKF parameter estimation algorithm to solve this TPBVP.

\section{UKF Parameter Estimation}

The parameter estimation problem, which is also known as the system identification problem, is a process which calculates the parameters of the system model according to the input and output data.

For a system model, it can be expressed by nonlinear mapping

$$
\boldsymbol{y}_{k}=G\left(\boldsymbol{x}_{k}, \boldsymbol{w}\right)
$$

Where $\boldsymbol{w}$ is a group of unknown parameters, $\boldsymbol{x}_{k}$ is the input data, and $\boldsymbol{y}_{k}$ is the output data. Generally speaking, the input data $\boldsymbol{x}_{k}$ and expected output data $\boldsymbol{d}_{k}$ is constant, the output error can be defined as $\boldsymbol{e}_{k}=\boldsymbol{d}_{k}-G\left(\boldsymbol{x}_{k}, \boldsymbol{w}\right)$. The purpose of parameter estimation problem is to calculate $\boldsymbol{w}$ and minimize $\boldsymbol{e}_{k}$. To estimate parameters by UKF, the original problem can be written as

$$
\left\{\begin{array}{l}
\boldsymbol{w}_{k+1}=\boldsymbol{w}_{k}+\boldsymbol{r}_{k} \\
\boldsymbol{d}_{k}=G\left(\boldsymbol{x}_{k}, \boldsymbol{w}_{k}\right)+\boldsymbol{e}_{k}
\end{array}\right.
$$

Where $\boldsymbol{r}_{k}$ is the process noise, $\boldsymbol{d}_{k}$ is the expected output, and $\boldsymbol{e}_{k}$ is the observation noise. Then the original parameter estimation problem can be solved by UKF[8].

In the view of optimization, the parameter estimation problem can be regarded as an optimization problem in which the parameters $\boldsymbol{w}$ are the optimization variables, that is $\min J(\boldsymbol{w})$, where

$$
J(\boldsymbol{w})=\sum_{t=1}^{k}\left[\boldsymbol{d}_{t}-G\left(\boldsymbol{x}_{t}, \boldsymbol{w}\right)\right]^{\mathrm{T}}\left(\boldsymbol{R}^{\mathrm{e}}\right)^{-1}\left[\boldsymbol{d}_{t}-G\left(\boldsymbol{x}_{t}, \boldsymbol{w}\right)\right]
$$

The output error can be defined as $\boldsymbol{q}_{k}=\boldsymbol{d}_{k}-G\left(\boldsymbol{x}_{k}, \boldsymbol{w}\right)$, thus the Eq. 15 can be rewritten in the form of observation error as follows

$$
\left\{\begin{array}{l}
\boldsymbol{w}_{k}=\boldsymbol{w}_{k-1}+\boldsymbol{r}_{k} \\
0=-\boldsymbol{q}_{k}+\boldsymbol{e}_{k}
\end{array}\right.
$$

Finally the original problem is converted into a parameter estimation problem in which the expected value of the observation error is 0 . 


\section{The TPBVP Solved by UKF}

To convert the lunar ascent TPBVP to the parameter estimation problem, firstly, set the initial value of costate variables to the parameters to be estimated, and we can conclude that $\lambda_{\theta}=0$ through transversality conditions and equations of state, so the parameters to be estimated are

$$
\boldsymbol{w}=\left[\lambda_{r}\left(t_{0}\right), \lambda_{v}\left(t_{0}\right), \lambda_{\omega}\left(t_{0}\right), \lambda_{m}\left(t_{0}\right)\right]^{\mathrm{T}}
$$

The observation error is

$$
q=c_{1}\left|\boldsymbol{r}_{\mathrm{f}}-\boldsymbol{r}\left(t_{\mathrm{f}}\right)\right|+c_{2}\left|\boldsymbol{r}_{\mathrm{f}} \boldsymbol{\omega}_{\mathrm{f}}-\boldsymbol{r}\left(t_{\mathrm{f}}\right) \boldsymbol{\omega}\left(t_{\mathrm{f}}\right)\right|+c_{3}\left|\boldsymbol{v}_{\mathrm{f}}-\boldsymbol{v}\left(t_{\mathrm{f}}\right)\right|
$$

Where $\boldsymbol{r}_{\mathrm{f}}, \boldsymbol{v}_{\mathrm{f}}$ and $\boldsymbol{\omega}_{\mathrm{f}}$ indicate the predetermined distance to the center of the Moon, radial velocity and angular velocity, respectively. $\boldsymbol{r}\left(t_{\mathrm{f}}\right), \boldsymbol{v}\left(t_{\mathrm{f}}\right)$ and $\boldsymbol{\omega}\left(t_{\mathrm{f}}\right)$ indicate the calculated terminal distance to the center of the Moon, radial velocity and angular velocity, respectively. $c_{1}, c_{2}$ and $c_{3}$ are the penalty factors.

Thus, the TPBVP is corresponding to the Eq. 17 in form and can be solved by the UKF parameter estimation algorithm. By adjusting the initial values of the costate variables constantly through the UKF parameter estimation algorithm and integrating the state and costate equations, the nominal trajectory which meets the requirement will be calculated.

Fig. 2 is the flow chart of the UKF parameter estimation algorithm.

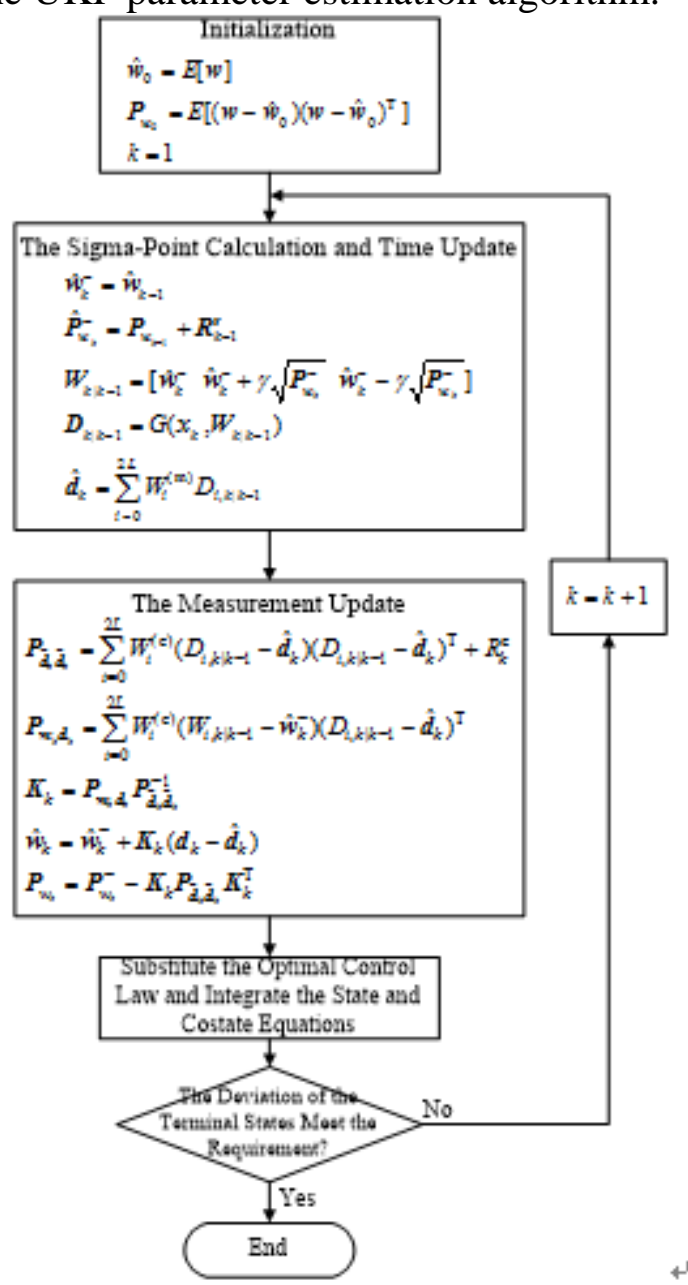

Figure 2 UKF parameter estimation flow-process graph

The parameters of the UKF parameter estimation algorithm are as follows 


$$
\left\{\begin{array}{l}
\lambda=\alpha^{2}(N+\kappa)-N \\
\gamma=\sqrt{N+\lambda} \\
W_{0}^{(\mathrm{m})}=\frac{\lambda}{N+\lambda} \\
W_{0}^{(\mathrm{c})}=\frac{\lambda}{N+\lambda}+1-\alpha^{2}+\beta \\
W_{i}^{(\mathrm{m})}=W_{i}^{(\mathrm{c})}=\frac{1}{2(N+\lambda)}, \quad i=1,2, \cdots, 2 N
\end{array}\right.
$$

Where $N$ is the dimension of $\boldsymbol{w}, \lambda$ is a scaling parameter. The constant $\alpha$ determines the spread of the sigma points around $\overline{\boldsymbol{w}}$, and is usually set to a small positive value (e.g. $\left.\left[10^{-4}, 1\right]\right)$. The constant $\kappa$ is a secondary scaling parameter to ensure the positive semi definiteness of the covariance matrices, which is usually set to $0 . \beta$ is used to incorporate prior knowledge of the distribution of $\boldsymbol{w}$ (for Gaussian distributions, $\beta=2$ is optimal). $W_{i}^{(\mathrm{m})}$ is the weight when calculate the mean, and $W_{i}^{(\mathrm{c})}$ is the weight when calculate the covariance. $\boldsymbol{R}^{\mathrm{r}}$ is the process-noise covariance, $\boldsymbol{R}^{\mathrm{e}}$ is the measurement-noise covariance.

\section{Numerical Simulation}

The Data for Simulation. The boundary conditions simulate the lunar ascent module to direct injection at the perigee of a $15 \times 100 \mathrm{~km}$ elliptical transfer orbit. The initial and terminal conditions are shown in Table 1, and constants for the simulation are provided in Table 2 (where $v_{\mathrm{T}}$ is the tangential velocity, and $t_{\mathrm{s}}$ is the simulation step).

Table 1 Boundary conditions

\begin{tabular}{lcc}
\hline Symbol & Initial Values & Terminal Values \\
\hline$r /[\mathrm{m}]$ & 1738000 & 1753000 \\
$v_{\mathrm{T}} /\left[\mathrm{m} \cdot \mathrm{s}^{-1}\right]$ & 0 & 1692.048 \\
$v /\left[\mathrm{m} \cdot \mathrm{s}^{-1}\right]$ & 0 & 0 \\
$\theta /\left[^{\circ}\right]$ & 0 & free \\
$m /[\mathrm{kg}]$ & 3000 & free \\
$t /[\mathrm{s}]$ & 0 & free \\
\hline
\end{tabular}

Table 2 Constants for the simulation

\begin{tabular}{lccc}
\hline Symbol & Quantity & Symbol & Quantit \\
& & & $\mathrm{y}$ \\
\hline$\mu_{\mathrm{L}} /[\mathrm{m}]$ & 1738000 & $I_{\mathrm{sp}} /(\mathrm{s})$ & 300 \\
$t_{\mathrm{s}} /[\mathrm{s}]$ & 1 & $F /(\mathrm{N})$ & 10000 \\
\hline
\end{tabular}

Simulation Results. Set the initial values of the costate variables to random values (e.g. 1), the optimal values calculated by the UKF parameter estimation algorithm are shown in Table 3.

Table 3 Initial and optimal values of costate variables

\begin{tabular}{ccc}
\hline Costate Variables & Initial Values & Optimal Values \\
\hline$\lambda_{r}$ & 1 & 0.0989 \\
$\lambda_{v}$ & 1 & 0.7116 \\
$\lambda_{\omega}$ & 1 & $4.0451 \times 10^{6}$ \\
$\lambda_{m}$ & 1 & 1.0000 \\
\hline
\end{tabular}


The simulation results of the lunar ascent nominal trajectory are shown in Fig. 3.
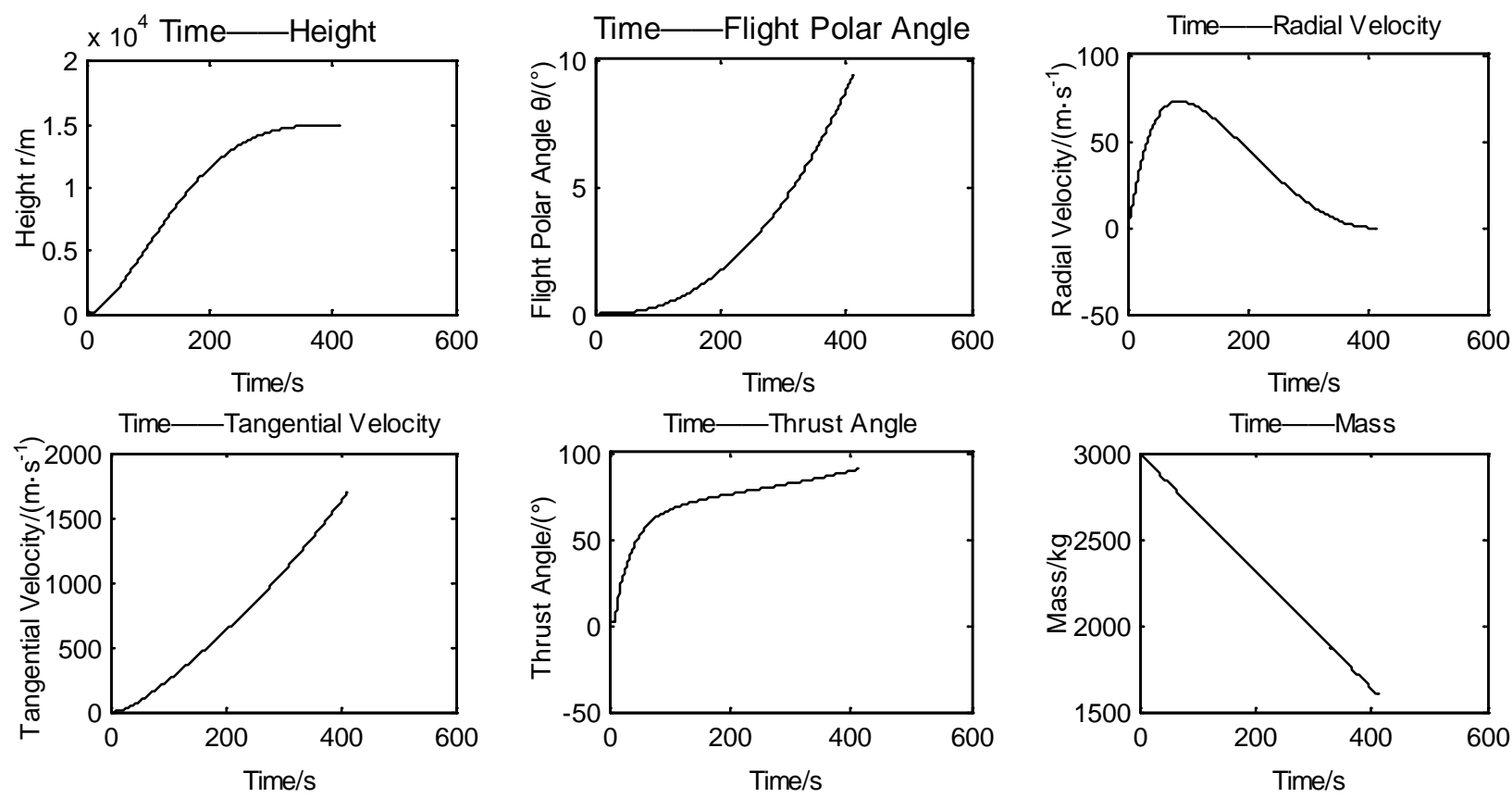

Fig 3 Curve graphs of state and control variables

The deviations between terminal states and desired states are provided in Table 4 .

Table 4 Terminal state deviations of simulation results

\begin{tabular}{cccc}
\hline State Variables & $\boldsymbol{r} /[\mathrm{m}]$ & $\boldsymbol{v}_{\mathrm{T}} /\left[\mathrm{m} \cdot \mathrm{s}^{-1}\right]$ & $\boldsymbol{v} /\left[\mathrm{m} \cdot \mathrm{s}^{-1}\right]$ \\
\hline Deviations & 0.51 & 4.570 & -0.0183 \\
\hline
\end{tabular}

The simulation results indicate that the change of the states and control variables are relatively smooth, and the deviations of the terminal states meet the constraint condition, which prove that the algorithm is feasible.

Contrast Simulation. In order to verify the rapidity of the algorithm, set the initial values of the costate variables to guessed values and solve this problem by the UKF parameter estimation algorithm, genetic algorithm (GA) and particle swarm optimization (PSO), respectively. Because GA and PSO are random algorithm, the simulation results are different each time. But solving by the UKF parameter estimation algorithm will get the same result when the covariance matrix and the initial values of the costate variables are unchanged. To avoid the randomness of the results, 100 times of simulation test are carried out using GA and PSO respectively, and set the initial values of the costate variables to 100 groups of random initial values and 1 group of guessed values respectively and then solved by the UKF parameter estimation algorithm. The contrast simulation results are shown in Table 5. The first 3 columns of data are mean of 100 times of simulation results, and the last column of data is a single simulation result.

Table 5 Comparison of the optimization results

\begin{tabular}{ccccc}
\hline & \multirow{2}{*}{ GA } & \multirow{2}{*}{ PSO } & \multicolumn{2}{c}{ UKF } \\
& & & Random Values & Guess Values \\
\hline Deviation of $\boldsymbol{r} /[\mathrm{m}]$ & 0.24 & 0.11 & 0.36 & 0.14 \\
Deviation of $\boldsymbol{v}_{\mathrm{T}} /\left[\mathrm{m} \cdot \mathrm{s}^{-1}\right]$ & 4.513 & 4.547 & 4.562 & 4.550 \\
Deviation of $\boldsymbol{v} /\left[\mathrm{m} \cdot \mathrm{s}^{-1}\right]$ & -0.0223 & -0.00426 & -0.0332 & -0.0263 \\
Fuel Consumption $/[\mathrm{kg}]$ & 1400.410 & 1400.410 & 1400.410 & 1400.410 \\
\hline
\end{tabular}

Convergence speed comparison speed is shown in Fig. 4. 


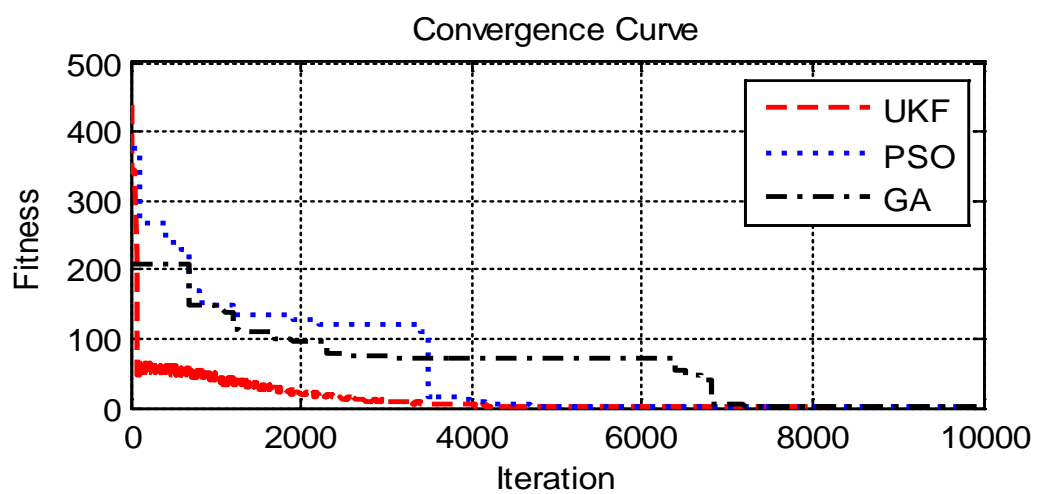

Figure 4 Convergence curve of UKF, PSO and GA

It can be concluded from Table 5 that when the deviations obtained by UKF parameter estimation algorithm are little higher than other algorithms based on random initial values. However, the Fig. 4 show that the convergence speed of the UKF parameter estimation is much higher than POS and GA, especially at the beginning of the optimization. Therefore, the UKF parameter estimation algorithm have better performance on computation efficiency.

\section{Summary and Conclusions}

The lunar ascent nominal trajectory requiring minimum fuel consumption has been solved by the UKF parameter estimation algorithm. The main advantages of this algorithm are the avoidance of deriving the gradient matrix and guessing the initial values of the costate variables, which are the main difficulties of the traditional indirect method. The numerical results show that it's more suited to optimize the lunar ascent nominal trajectory than other traditional optimization algorithms. Further study will focus on using 3-D system model, taking more control variables into account and optimizing the parameter configuration of the UKF algorithm.

\section{References}

[1] L. T. Biegler. An Overview of Simultaneous Strategies for Dynamic Optimization, Chemical Engineering and Processing:Process Intensification, Vol. 46, No. 11,2007,pp.1043-1053.

[2] Tongren Liu. A calculation of optimal flight trajectory using the parameterized optimization method, Acta Aeronautica et Astronautica Sinica, Vol. 15,No. 11,1994,pp.1298-1305.

[3] Wangwang Liu, Weiren Wu, Shengying Zhu, et al. 3-Dimensional lunar ascent reference trajectory optimization and analyses via gauss pseudospectral method, Proceedings of the 2011 Chinese Control and Decision Conference, Mianyang:IEEE,2011,pp.219-224.

[4] Guoqianq Huang, Yuping Lu, Ying Nan. A survey of numerical algorithms for trajectory optimization of flight vehicles, Sci China Tech Sci, Vol. 42, No. 9, 2012,pp.1016-1036.

[5] Pengfei Meng. Research on reentry target tracking and positioning and trajectory prediction, Chengdu: University of Electronic Science and Technology of China, 2013.

[6] Jian Li, Chao Han. Unscented Kalman filter for low-thrust orbit transfer optimization, Journal of Astronautics, Vol.35, No.2, 2014, pp.144-150.

[7] D. K. Larry, P. P. Tara. Altair descent and ascent reference trajectory and initial dispersion analyses, AIAA Guidance, Navigation and Control Conference, Toronto, Canada, August 2-5, 2010.

[8] S. Haykin. Kalman filtering and neural networks, John Wiley \& Sons Inc, 2002. 